\title{
Long non-coding RNA TPT1-AS1 alleviates cell injury and promotes the production of extracellular matrix by targeting the microRNA-324-5p/CDK16 axis in human dermal fibroblasts after thermal injury
}

\author{
JINHUA LUO*, HAOYUAN YOU*, JIANHUA ZHAN, GUANGHUA GUO, XING CHENG and GUOYU ZHENG
}

Department of Burns, The First Affiliated Hospital of Nanchang University, Nanchang, Jiangxi 330006, P.R. China

Received October 9,2020; Accepted April 30, 2021

DOI: $10.3892 /$ etm.2021.10275

\begin{abstract}
Long non-coding RNAs (lncRNAs) are associated with the healing of burn wounds in the dermis. The present study aimed to probe the role and regulatory network of the IncRNA TPT1 antisense RNA 1 (TPT1-AS1) in human dermal fibroblasts (HDFs) following thermal injury. A model of thermally injured cells was constructed with HDFs. The levels of TPT1-AS1, microRNA (miR)-324-5p and cyclin-dependent kinase (CDK)16 were determined through reverse transcription-quantitative PCR. Cell viability, cell cycle distribution, cell apoptosis rate and extracellular matrix (ECM) synthesis were assessed with a series of in vitro gain-of-function experiments and MTT, flow cytometry and western blot analyses. The binding ability of miR-324-5p and TPT1-AS1 (or the 3' untranslated region of CDK16) was identified via bioinformatics analysis and luciferase reporter assay. It was found that TPT1-AS1 and CDK16 were downregulated, but miR-324-5p was upregulated, in the HDFs after thermal injury. TPT1-AS1 elevation induced cell viability and ECM synthesis but attenuated cell cycle arrest at the $\mathrm{G}_{0} / \mathrm{G}_{1}$ stage and decreased the cell apoptosis rate of thermally injured HDFs. In addition, TPT1-AS1 sponged miR-324-5p to modulate CDK16 expression. Moreover, silencing CDK16 weakened the impacts of TPT1-AS1 upregulation on cell function and ECM synthesis in heat-treated HDFs. In summary, TPT1-AS1 relieved cell injury and induced ECM synthesis by sponging miR-324-5p and targeting CDK16 in the HDFs after thermal injury, implying a protective role for TPT1-AS1 in the burn wound healing process.
\end{abstract}

Correspondence to: Dr Jianhua Zhan, Department of Burns, The First Affiliated Hospital of Nanchang University, 17 Yongwaizheng Street, Nanchang, Jiangxi 330006, P.R. China

E-mail: zhanjh972@hotmail.com

*Contributed equally

Key words: TPT1 antisense RNA 1, microRNA-324-5p, CDK16, thermal injury

\section{Introduction}

Burn wound healing can be characterized as overheating, manifested by hypertrophic scar formation after burn injury (1). The treatment of deep burn wounds is a great challenge due to scar formation and poor functioning after wound healing (2). The proliferation of human dermis fibroblasts (HDFs) induced in the dermis exerts a crucial effect on burn wound repair $(2,3)$ and maintains the extracellular matrix (ECM) synthesis to elevate scar quality $(2,4)$. When skin is injured, HDFs are activated to restore tissue integrity. ECM metabolism is the major factor of burn wound repair quality and outcomes (5). However, the regulatory mechanism in the burn wound healing process has not been specifically reported.

Long non-coding RNAs (lncRNAs) are classified as a group of important regulators involved in multiple biological processes (6-8). As reported, IncRNAs exert vital effects on wound healing after thermal injury. For instance, the IncRNA LET induces cell proliferative ability and suppresses fibroblast apoptosis during burn wound healing (9). Additionally, the lncRNA AC067945.2 represses collagen levels and promotes ECM synthesis by fibroblasts in the wound healing process (10). According to the literature, a microarray analysis illustrated that TPT1-AS1 is downregulated in denatured dermal tissues (3). However, there has been no report on how TPT1-AS1 exerts its regulatory function in burn wound healing. Thus, the aim of the present study was to investigate the biological role of TPT1-AS1 in HDFs after thermal injury.

MicroRNAs (miRNAs) are small endogenous RNAs that regulate gene expression post-transcriptionally $(11,12)$. In addition, IncRNAs exert theirinfluences by serving as competing endogenous RNAs (ceRNAs) for miRNAs to derepress messenger RNAs (mRNAs) (13). For example, the lncRNA ZFAS1 regulates proliferation, apoptosis and the inflammatory response via the miR-2682-5p/ADAMTS9 axis in rheumatoid arthritis (14). Furthermore, the MEG3/miR-93/Nrf2 axis represses high glucose-induced apoptosis in retinal pigment epithelium cells (15). Hence, whether TPT1-AS1 exerts its regulatory function via the ceRNA pattern in the burn wound healing process was investigated in the present study.

The present study primarily focused on the biological function of TPT1-AS1 in HDFs after burn injury. It was 
hypothesized that TPT1-AS1 may exert its regulatory effect as a ceRNA in HDFs by interacting with miRNA. The findings revealed that TPT1-AS1 mitigated cell injury and promoted ECM synthesis by interacting with microRNA 324-5p (miR-324-5p) and targeting cyclin-dependent kinase 16 (CDK16) in HDFs after thermal injury. The present study findings may provide a potential novel insight for clinical burn wound treatment.

\section{Materials and methods}

Cell culture and treatment. Human dermal fibroblasts $\left(\right.$ ATCC $^{\circledR}$, PCS-201-012 ${ }^{\mathrm{TM}}$ ) were provided by American Type Culture Collection. and cultured in RPMI-1640 medium (Gibco; Thermo Fisher Scientific, Inc.) containing $10 \%$ fetal bovine serum (FBS; Gibco; Thermo Fisher Scientific, Inc.) in an incubator with a $5 \% \mathrm{CO}_{2}$ atmosphere at $37^{\circ} \mathrm{C}$. The use of HDFs was approved by the ethics committee of The First Affiliated Hospital of Nanchang University before the study. To establish a cellular heat-injury model, HDFs were challenged in $52^{\circ} \mathrm{C}$ water for $30 \mathrm{sec}(2,9,16)$, while cells in the control group were maintained in $37^{\circ} \mathrm{C}$ water for the same duration. In succession, cells were cultivated in the incubator at normal temperatures.

Cell transfection. For cell transfection, short harpin RNA (shRNA) against CDK16 (sh-CDK16\#1, CCACUGAGGACA UCAACAA; and sh-CDK16\#2, GGAGAUCAGACUGGA ACAU), a negative control (sh-NC; scrambled; AGCCUA ACCUAGAACACAG), the TPT1-AS1 overexpression vector (pcDNA3.1-TPT1-AS1), an empty vector (pcDNA3.1-empty), miR-324-5p mimics (miR-324-5p), and miRNA negative control (NC mimics, scrambled) were synthesized by Shanghai GenePharma Co., Ltd. Using LipoFiter ${ }^{\mathrm{TM}}$ liposomal transfection reagent (Hanbio Biotechnology Co., Ltd.), HDFs were transfected with $40 \mathrm{nM}$ sh-CDK16\#1/2, $10 \mathrm{nM}$ miR-324-5p mimic or $2 \mu \mathrm{g}$ pcDNA3.1 vector upon reaching $60-70 \%$ confluence in 6-well plates. Twenty-four hours after transfection, the cells were collected for thermal injury or further analysis.

Reverse transcription-quantitative polymerase chain reaction $(R T-q P C R)$. Total RNA was extracted with TRIzol ${ }^{\circledR}$ reagent (Thermo Fisher Scientific, Inc.), and the concentration and purity were assessed with a NanoDrop ND-2000 spectrophotometer (Thermo Fisher Scientific, Inc.). The RNA was reverse transcribed to cDNA with Taqman ${ }^{\mathrm{TM}}$ miRNA or a mRNA reverse transcription kit (Thermo Fisher Scientific, Inc.). The reaction conditions were as follows: $37^{\circ} \mathrm{C}$ for $25 \mathrm{~min}$, followed by $85^{\circ} \mathrm{C}$ for $5 \mathrm{~min}$. SYBR ${ }^{\circledR}$ Premix ExTaq ${ }^{\mathrm{TM}}$ II kit (Takara Bio, Inc.) was used to perform the PCR analysis on an ABI 7500 Real-Time PCR System. The thermocycling conditions were: Pre-denaturation at $90^{\circ} \mathrm{C}$ for $3 \mathrm{~min}$, denaturation at $90^{\circ} \mathrm{C}$ for $20 \mathrm{sec}$, annealing at $60^{\circ} \mathrm{C}$ for $20 \mathrm{sec}$, and extension at $72^{\circ} \mathrm{C}$ for $40 \mathrm{sec}$, for a total of 40 cycles. The samples were prepared in duplicate. GAPDH or U6 was used as the internal reference. The relative expression levels were calculated with the $2^{-\Delta \Delta \mathrm{Cq}}$ method (17). The following primer sequences were used: TPT1-AS1 forward, 5'-CTATCCTTG CCCATCTTCCT-3'; TPT1-AS1 reverse, 5'-TCTACCGGA GCAATTGGAG-3'; miR-324-5p forward, 5'-TCGGCAGGC
GCAUCCCCUAG-3'; miR-324-5p reverse, 5'-CACTCAACT GGTGTCGTGGA-3'; CDK16 forward, 5'-CTCTGCACCAGA GATTGTG-3', CDK16 reverse, 5'-CATACGCACTCTCAC TGGA-3'; GAPDH forward, 5'-TCATTTCCTGGTATGACA ACGA-3'; GAPDH reverse, 5'-GGTCTTACTCCTTGGAGG C-3'; U6 forward, 5'-CAATACAGAGAAAGTTAGCACG-3', and U6 reverse, 5'-AATGCTTCAAAGAGTTGTGC-3'.

3-(4,5-Dimethyl-2-thiazolyl)-2,5-diphenyl-2-H-tetrazolium bromide (MTT) assay. Primary HDFs were seeded at a density of $1 \times 10^{4}$ cells/well in 96-well plates and maintained for $24 \mathrm{~h}$ for cell transfection. The viability of the transfected cells and non-transfected cells was measured by MTT assay 24, 48 and $72 \mathrm{~h}$ post-transfection. The optical density at $490 \mathrm{~nm}$ was measured and utilized to plot a proliferation curve.

Flow cytometric analysis. For the cell cycle analysis, trypsin-digested primary HDFs were seeded into 6-well plates at a density of $5 \times 10^{4}$ cells/well and cultured for $24 \mathrm{~h}$. The HDFs were then harvested, centrifuged for $5 \min \left(4^{\circ} \mathrm{C} ; 1,000 \mathrm{x} \mathrm{g}\right)$, and fixed with $70 \%$ ethanol at $4^{\circ} \mathrm{C}$ for $24 \mathrm{~h}$. The fixed HDFs were treated with propidium iodide (PI; Clontech Laboratories, Inc.) at room temperature for $30 \mathrm{~min}$. Finally, the cell cycle was evaluated with a FACSCanto flow cytometer and CellQuest software version 5.1 (both obtained from BD Biosciences). The assays were performed in three triplicates. For the cell apoptosis analysis, HDFs $\left(1 \times 10^{5}\right)$ were seeded in 24 -well plates and cultivated for $24 \mathrm{~h}$. Annexin V-fluorescein isothiocyanate (FITC) and PI (Clontech Laboratories, Inc.) were added to the cell cultures and incubated at room temperature for $10 \mathrm{~min}$ in the dark. Finally, the FACSCanto flow cytometer was used to assess the cell apoptosis rate. Annexin V-positive and PI-negative cells were considered as apoptotic cells.

Western blot analysis. Total protein was extracted with RIPA lysis buffer (cat. no. R0020; Beijing Solarbio Science \& Technology Co., Ltd.) after the HDFs were washed with PBS. After high-speed centrifugation at $1,000 \mathrm{x}$ g for $10 \mathrm{~min}$ at $4^{\circ} \mathrm{C}$, the bicinchoninic acid (BCA) method was used to quantify the protein in the supernatant using a BCA protein quantification kit (Shanghai Yeasen Biotechnology Co., Ltd.). An equal amount of protein $(20 \mu \mathrm{g})$ was prepared for $10 \%$ SDS-PAGE, after which the proteins were transferred to a nitrocellulose membrane (EMD Millipore) with Western transfer buffer (Beyotime Institute of Biotechnology). Each sample was prepared in triplicate. The membranes were blocked with QuickBlock ${ }^{\mathrm{TM}}$ blocking buffer (Beyotime Institute of Biotechnology) at room temperature for $15 \mathrm{~min}$, allowed to interact overnight with primary antibodies against cyclin A1 (cat. no. ab53699; 1:500), cyclin D1 (cat. no. ab16663; 1:200), cyclin E1 (cat. no. ab33911; 1:1,000), CDK4 (cat. no. ab108357; 1:1,000), Bcl-2 (cat. no. ab32124; 1:1,000), Bax (cat. no. ab32503; 1:1,000), cleaved caspase-3 (cat. no. ab13847; 1:500), collagen I (cat. no. ab34710; $1: 1,000), \alpha$-smooth muscle actin ( $\alpha$-SMA; cat. no. ab7817; 1:3,000), CDK16 (cat. no. ab181208; 1:1,000) or GAPDH (cat. no. ab8245; 1:500) (all from Abcam) at $4^{\circ} \mathrm{C}$ and then cultured with IgG H\&L (HRP) secondary antibody (cat. no. ab205718; 1:2,000) for $2 \mathrm{~h}$. The antibodies were all purchased from Abcam. BeyoECL Plus (Beyotime Institute of Biotechnology) and film (Carestream Health, Inc.) were 
applied to visualize the western blots in the dark. Quantum One software version 4.6.8 (Bio-Rad Laboratories, Inc.) was used to analyze the relative protein levels using GAPDH as an internal control.

Bioinformatics analysis. The subcellular localization of the IncRNA TPT1-AS1 was predicted by the 1ncLocator website (http://www.csbio.sjtu.edu.cn/bioinf/lncLocator/). Additionally, the binding site between TPT1-AS1 and miR-324-5p was acquired through 'StarBase' (http://starbase. sysu.edu.cn). The binding site of miR-324-5p in the 3' untranslated region (3'UTR) of CDK16 was obtained from TargetScan (http://www.targetscan.org/vert_72/).

Subcellular fractionation assay. Cytoplasmic and nuclear extracts were obtained from HDFs by Invitrogen PARIS nuclear and cytoplasmic extraction kit (cat. no. AM1921; Invitrogen; Thermo Fisher Scientific, Inc.). RNA isolated from the nucleus or cytoplasm using the Invitrogen PARIS kit (cat. no. AM1921; Invitrogen; Thermo Fisher Scientific, Inc.) was subjected to RT-qPCR analysis. The levels of U6 (nuclear control), GAPDH (cytoplasmic control), and TPT1-AS1 were determined.

Luciferase reporter assay. For the luciferase reporter assay, wild-type (Wt) or mutant (Mut) luciferase reporter vectors TPT1-AS1 (or the 3'UTR of CDK16) were established in firefly luciferase-expressing pmirGLO vector (Promega Corporation), termed TPT1-AS1-Wt, TPT1-AS1-Mut, CDK16-Wt and CDK16-Mut, respectively. HDFs were co-transfected with the $50 \mathrm{nM}$ of miR-324-5p mimics or NC mimics, $200 \mathrm{ng}$ of $\mathrm{Wt}$ or Mut luciferase reporter constructs and a Renilla vector using LipoFiter $^{\mathrm{TM}}$ liposomal transfection reagent. Forty-eight hours after transfection, the luciferase activity was measured with a luciferase reporter assay kit (Promega Corporation).

Statistical analysis. Statistical analysis was performed using GraphPad Prism 7 software (GraphPad Software, Inc.), and the data are expressed as the means \pm standard deviation (S.D.). All experiments were repeated three times. Differences between two groups were assessed for significance by Student's t test, and differences of multiple groups were assessed by ANOVA followed by Tukey's post hoc test. $\mathrm{P}<0.05$ was considered to indicate a statistically significant difference.

\section{Results}

TPT1-AS1 relieves cell injury and induces ECM synthesis in thermally injured HDFs. Firstly, TPT1-AS1 expression level was measured in HDFs under heat stimulation. According to the RT-qPCR results, the levels of TPT1-AS1 were decreased after thermal injury in HDFs (Fig. 1A). Subsequently, the transfection efficiency of TPT1-AS1 in HDFs was confirmed using RT-qPCR analysis (Fig. 1B). In order to investigate the biological role of TPT1-AS1 in HDFs after thermal injury, the efficacy of the TPT1-AS1 transfection and overexpression in HDFs was verified (Fig. 1C). MTT assays showed that TPT1-AS1 overexpression reversed the thermal injury-induced decrease in HDF viability (Fig. 1D). The flow cytometry results suggested that thermal injury caused a significant increase in the proportion of cells at the $\mathrm{G}_{0} / \mathrm{G}_{1}$ stage and a decrease in the proportion of cells at the $\mathrm{S}$ stage, which indicated that thermal injury induced cell cycle arrest at the $\mathrm{G}_{0} / \mathrm{G}_{1}$ stage . The upregulation of TPT1-AS1 evidently attenuated cycle arrest at the $G_{0} / G_{1}$ phase (Fig. 1E). The western blot analysis showed that the levels of cyclin A1, cyclin D1, cyclin E1 and CDK4 were significantly decreased in the heated HDFs, while TPT1-AS1 overexpression increased the levels of cell cycle-associated factors (Fig. 1F). The flow cytometry analysis suggested that the apoptosis rate of the HDFs was increased by thermal injury and then significantly suppressed by TPT1-AS1 overexpression (Fig. 1G-H). Furthermore, the western blot analysis demonstrated that the protein levels of Bax and cleaved caspase- 3 were significantly higher, whereas the level of Bcl-2 was decreased in the heated HDFs. However, TPT1-AS1 overexpression reversed the changes in the levels of apoptosis-associated proteins in thermally injured HDFs (Fig. 1I). Additionally, the western blot analysis revealed that, in terms of ECM synthesis, the heat stimulation-induced decrease in the protein levels of $\alpha$-SMA and collagen I were recovered by the overexpression of TPT1-AS1 in the HDFs (Fig. 1J).

TPT1-AS1 is a sponge of miR-324-5p. In order to further probe the regulatory mechanism of TPT1-AS1, the subcellular localization of TPT1-A1 was predicted with 1ncLocator. The results showed the cytoplasmic localization of TPT1-AS1 (Fig. 2A). A subcellular fractionation assay also validated the cytoplasm as the primary location of TPTI-AS1, which indicated TPT1-AS1 as a post-transcriptional regulator of gene expression (Fig. 2B). Then, miR-324-5p was screened as a potential downstream target of TPT1-AS1 with the 'StarBase' database (conditions; Low clip and low degradome) and verified its high expression in thermally injured HDFs via RT-qPCR (Fig. 2C). Subsequently, the miR-324-5p overexpression efficiency was verified in the HDFs (Fig. 2D). Moreover, the transfection efficiency of miR-324-5p mimics in heated HDFs was also confirmed by RT-qPCR analysis (Fig. 2E). The binding sequence of miR-324-5p in TPT1-AS1 was predicted. A luciferase reporter assay was performed to validate the binding capacity of miR-324-5p and TPT1-AS1. miR-324-5p upregulation decreased the luciferase activity of TPT1-AS1-Wt in the HDFs, while no evident change was observed for the TPT1-AS1-Mut group (Fig. 2F).

CDK16 is directly targeted by miR-324-5p. In order to further verify the hypothesis of the ceRNA regulatory pattern, 3 mRNAs (CDK16, KLF7 and SEPT3), were screened through overlapping prediction results of the microT, PicTar and TargetScan databases (Table SI). The RT-qPCR results illustrated that only CDK16 was significantly downregulated in the HDFs in response to miR-324-5p overexpression (Fig. 3A). The western blotting results showed that CDK16 protein levels were decreased by upregulating miR-324-5p (Fig. 3B). The target fragment of miR-324-5p at the 3'UTR of CDK16 is shown in Fig. 3C. A luciferase reporter experiment showed that the luciferase activity of the wild-type CDK16 3'UTR vector was significantly decreased in the presence of the miR-324-5p mimics, while no evident change was shown in the luciferase activity of the mutant CDK16 3'UTR (Fig. 3D). Furthermore, 

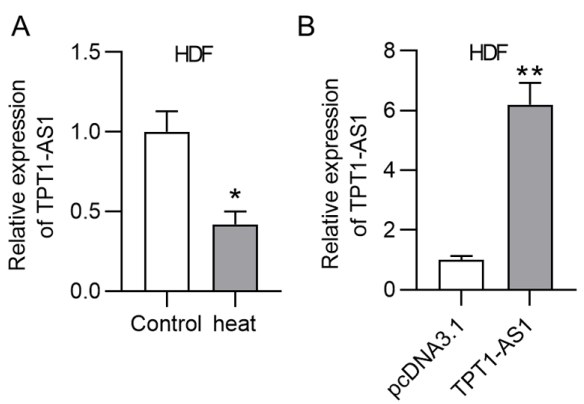
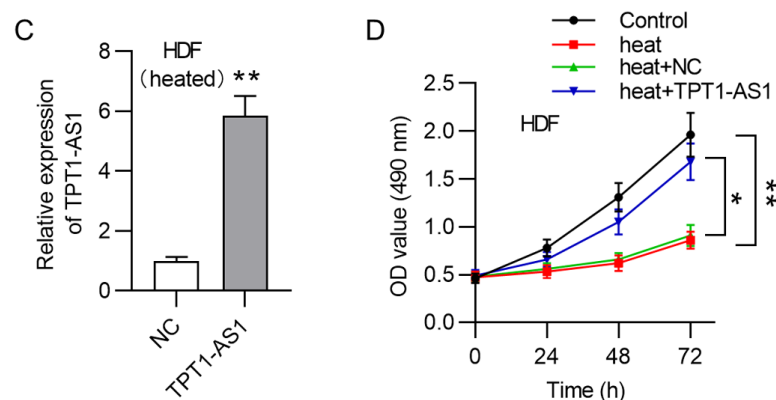

E
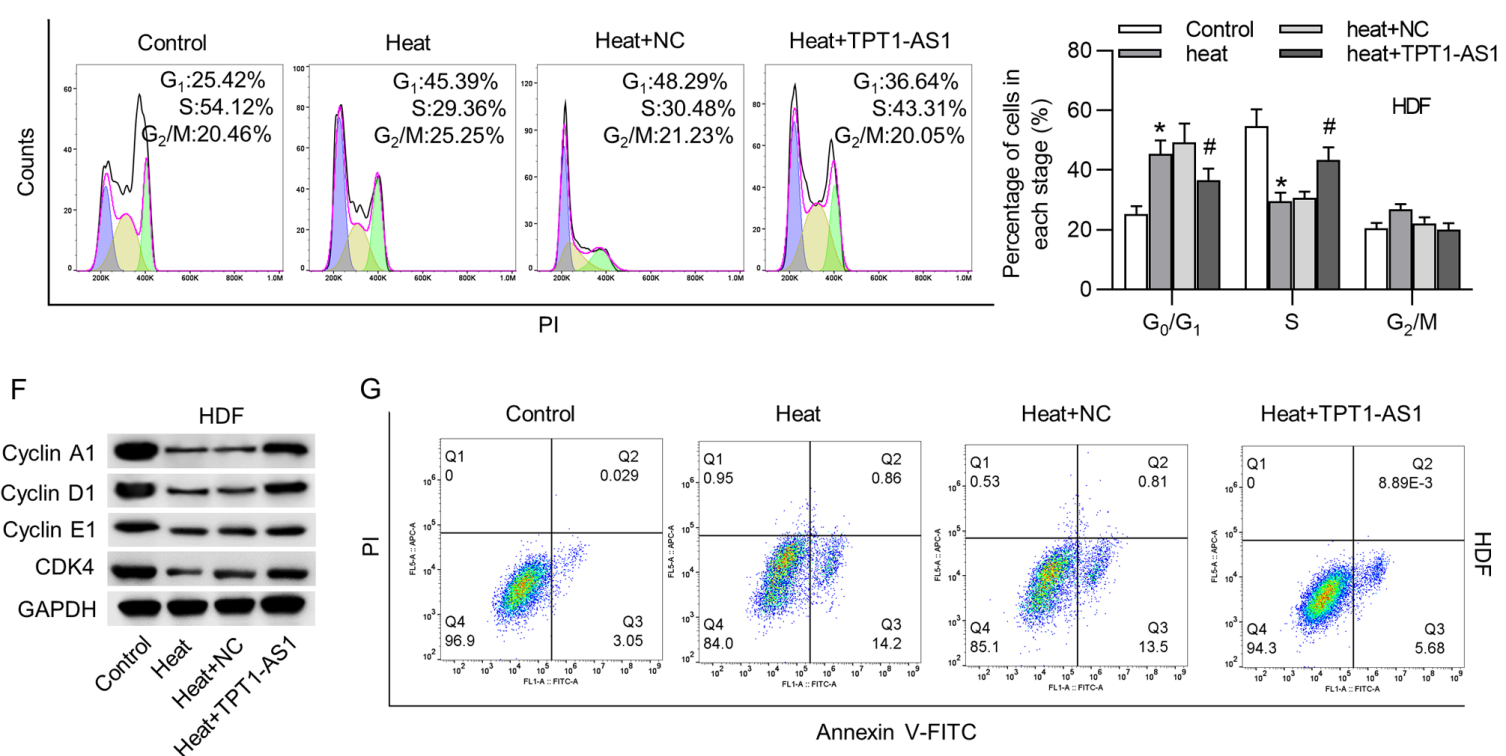

G
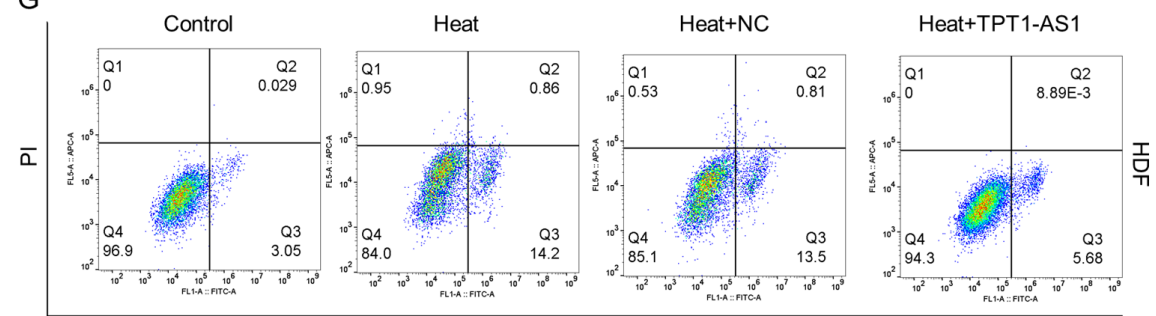

Annexin V-FITC

$\mathrm{H}$

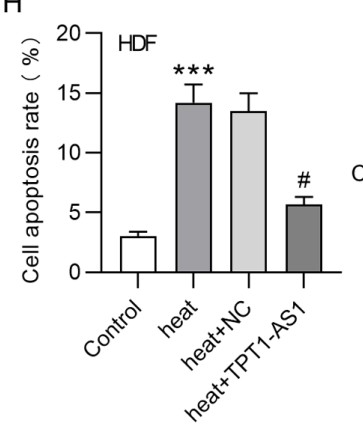

।

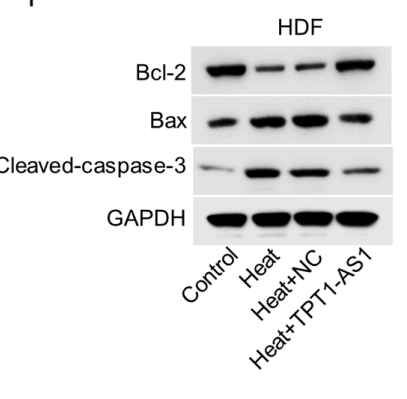

$J$

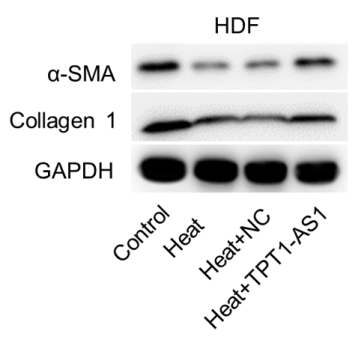

Figure 1. TPT1-AS1 is expressed at low levels and attenuates cell cycle arrest and apoptosis, but promotes ECM synthesis in the HDFs after thermal injury. (A) RT-qPCR was used to measure TPT1-AS1 levels in HDFs after thermal injury. (B) The overexpression efficiency of TPT1-AS1 in HDFs alone was determined using RT-qPCR. (C) RT-qPCR detected TPT1-AS1 expression level in the HDFs after heat stimulation. (D) MTT assay of HDF viability during TPT1-AS1 overexpression and heating. (E) Cell cycle progression was examined by flow cytometry after TPT1-AS1 overexpression in thermally injured HDFs. (F) Levels of cell cycle-associated proteins were verified in heated HDFs via western blot analysis. (G and H) Flow cytometry confirmed the apoptosis rate of the HDFs with TPT1-AS1 overexpression after thermal injury. (I) Western blot analysis validated the levels of apoptosis-associated markers in the heat-stimulated HDFs with upregulated TPT1-AS1. (J) Levels of ECM synthesis-associated proteins in the heat-treated HDFs were analyzed by western blot analysis after TPT1-AS1 upregulation. ${ }^{*} \mathrm{P}<0.05 ;{ }^{* *} \mathrm{P}<0.01 ;{ }^{* * *} \mathrm{P}<0.001 ;{ }^{*} \mathrm{P}<0.05$. TPT1-AS1, TPT1-antisense RNA 1; RT-qPCR, reverse transcription-quantitative PCR; ECM, extracellular matrix; HDFs, human dermal fibroblasts; NC, negative control.

CDK16 was expressed at low levels in the heat-stimulated HDFs according to the RT-qPCR results (Fig. 3E). A luciferase reporter assay also demonstrated that no evident change in the luciferase activity of the CDK16 promoter after miR-324-5p was overexpressed (Fig. 3F).

TPT1-AS1 alleviates cell injury and promotes ECM synthesis via the miR-324-5p/CDK16 axis in thermally injured HDFs. The knockdown efficiency of CDK16 in HDFs was verified (Fig. 4A). The expression of CDK16 was detected using
RT-qPCR analysis. The results showed that CDK16 expression was increased under TPT1-AS1 overexpression and then knocked down by the co-transfection of sh-CDK16\#1/\#2 (Fig. 4B). Furthermore, CDK16 knockdown rescued the pcDNA3.1/TPT1-AS1-mediated increase in the viability of the heat-treated HDFs (Fig. 4C). Flow cytometry revealed that TPT1-AS1 attenuated cycle arrest at the $\mathrm{G}_{0} / \mathrm{G}_{1}$ phase and caused a decrease in the proportion of cells at the $S$ stage, which were reversed by CDK16 silencing (Fig. 4D). Moreover, knocking down CDK16 reversed the inhibition 
A

\begin{tabular}{|l|l|}
\hline Subcellular locations & score \\
\hline Cytoplasm & 0.462476129497 \\
\hline Nucleus & 0.0470794562462 \\
\hline Ribosome & 0.157188420574 \\
\hline Cytosol & 0.191550876464 \\
\hline Exosome & 0.14170511722 \\
\hline
\end{tabular}

E

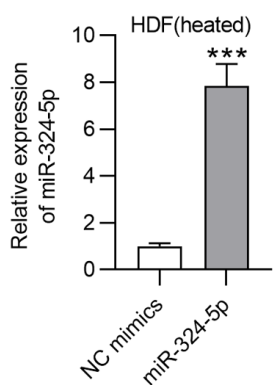

B

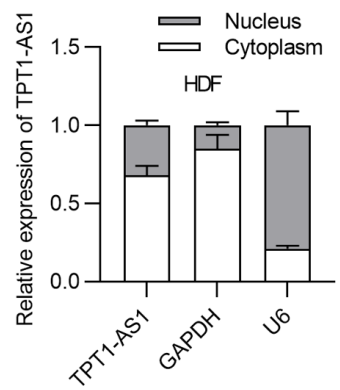

C

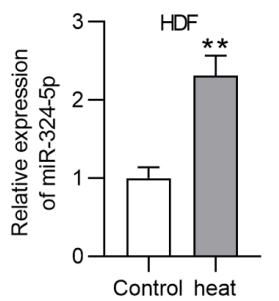

D

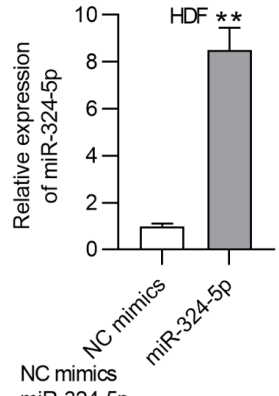

TPT1-AS1-Mut: $\quad 5^{\prime}$ cuAACACUGCAAAGUUUGCCCUACa 3'

TPT1-AS1-Wt: 5 ' CUAACACUGCAAAGUUUGGGAUGCa 3 miR-324-5p: $\quad 3$ ' ugUGGUUACG -- GGAUCCCCUACGC 5'

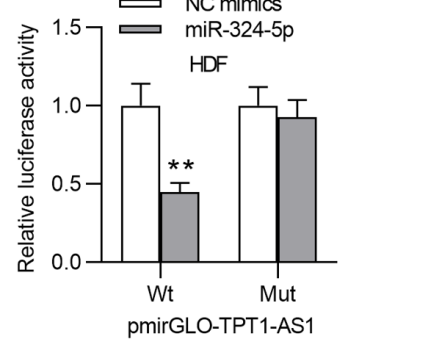

Figure 2. TPT1-AS1 binds to miR-324-5p. (A) Bioinformatics analysis predicted TPT1-AS1 subcellular localization in HDFs. (B) Subcellular fractionation assay revealed TPT1-AS1 localization in the HDFs. (C) miR-324-5p was predicted via 'StarBase', and its expression in heated HDFs was examined with RT-qPCR. (D) RT-qPCR was used to measure the transfection efficacy of miR-324-5p mimics in HDFs alone. (E) The overexpression efficiency of miR-324-5p was measured using RT-qPCR analysis in heated HDFs. (F) The binding ability of TPT1-AS1 and miR-324-5p was predicted with 'StarBase' and identified through a luciferase reporter assay. ${ }^{* *} \mathrm{P}<0.01 ;{ }^{* * *} \mathrm{P}<0.001$. miR, microRNA; TPT1-AS1, TPT1-antisense RNA 1; HDFs, human dermal fibroblasts; RT-qPCR, reverse transcription-quantitative PCR; Mut, mutant; Wt, wild-type; NC, negative control.

A

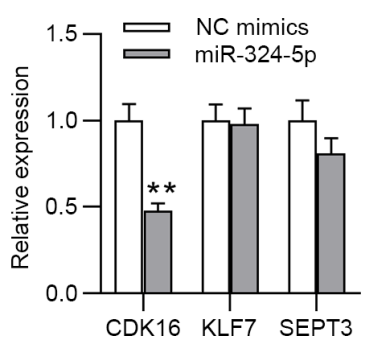

$\mathrm{E}$

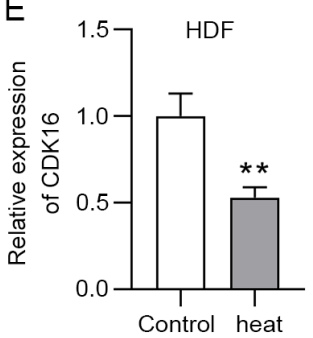

B

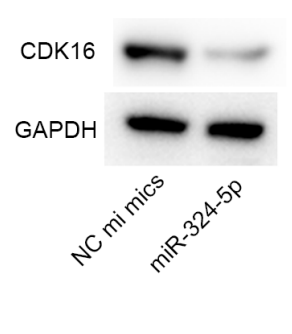

$\mathrm{F}$

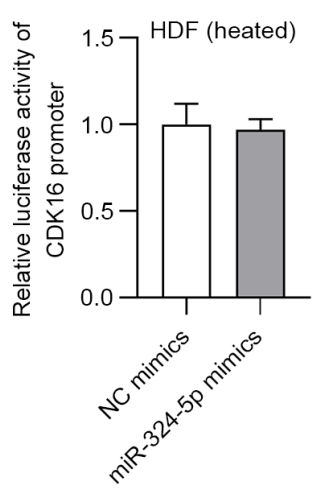

C

Position 36-42 of CDK16 3 ' UTR

CDK16-Mut: 5' caggCAGCGGCUGGACCCUACCc 3'

CDK16-Wt: 5' caggCAGCGGCUGGAGGGAUGCc 3'

miR-324-5p: 3' ugug GUUACGGGAUCCCCUACG c 5'
D

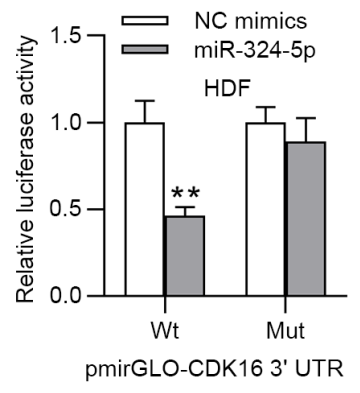

Figure 3. CDK16 is a target of miR-324-5p. (A) Putative mRNAs were obtained via bioinformatics analysis, and RT-qPCR was used to measure the expression of candidate mRNAs in response to miR-324-5p mimics. (B) Western blot analysis of the protein level of CDK16 after miR-324-5p upregulation. (C) The target sequence of miR-324-5p in the 3'UTR of CDK16 was obtained from TargetScan. (D) A luciferase reporter experiment verified the binding ability of CDK16 and miR-324-5p. (E) RT-qPCR analysis of CDK16 expression levels in HDFs after thermal injury. (F) A luciferase reporter assay was used to detect the luciferase activity of the CDK16 promoter after HDF transfection with miR-324-5p mimics. " $\mathrm{P}<0.01$. CDK16, cyclin-dependent kinase 16; miR, microRNA; RT-qPCR, reverse transcription-quantitative PCR; UTR, untranslated region; NC, negative control; HDFs, human dermal fibroblasts; Mut, mutant; Wt, wild-type.

of TPT1-AS1 overexpression on cell cycle-associated proteins in the thermally injured HDFs (Fig. 4E). CDK16 silencing counteracted the suppressive effect of TPT1-AS1 overexpression on the apoptosis of HDFs upon heat stimulation 
A

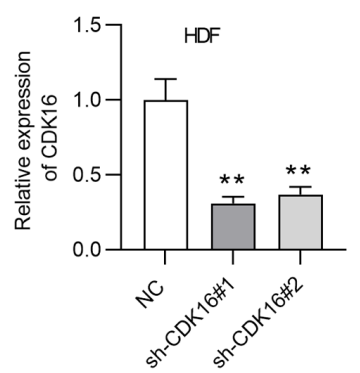

B

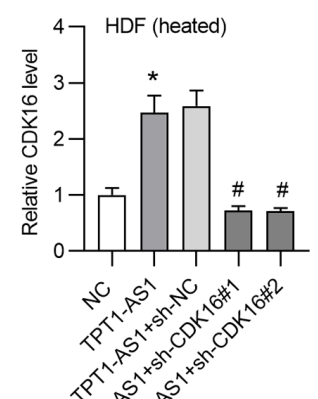

C

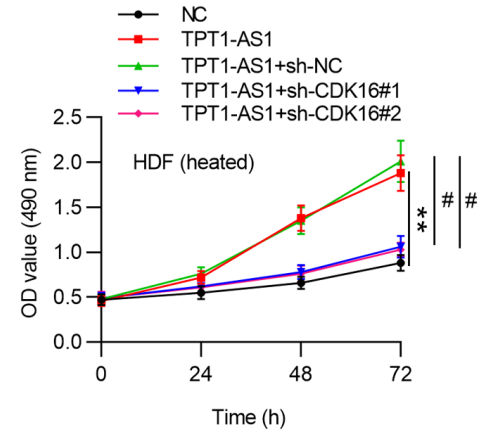

D

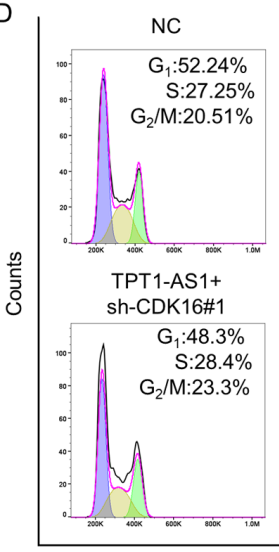

\section{TPT1-AS1}

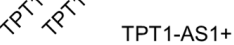

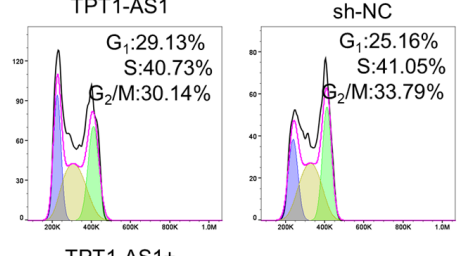

TPT1-AS1+ sh-CDK16\#2

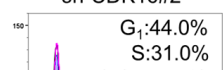

S:31.0\%

A

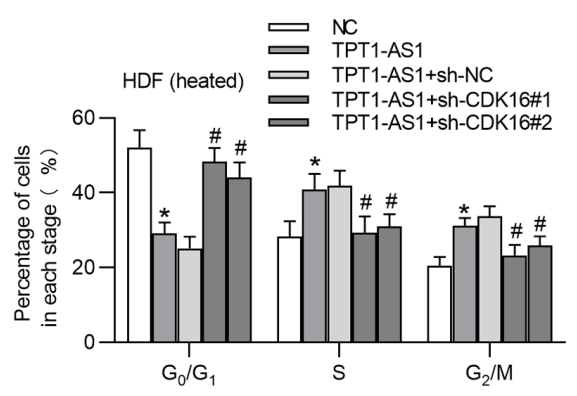

$\mathrm{PI}$

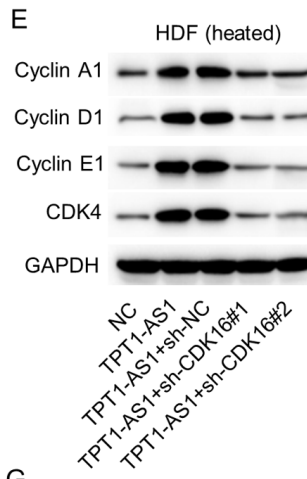

G

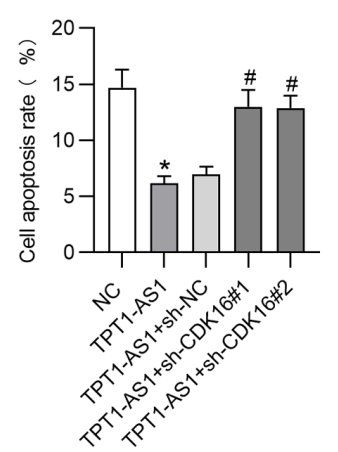

$\mathrm{F}$

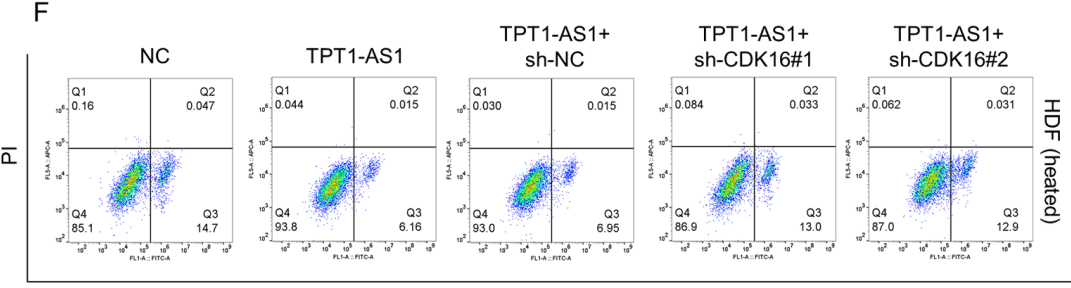

Annexin V-FITC

$\mathrm{H}$

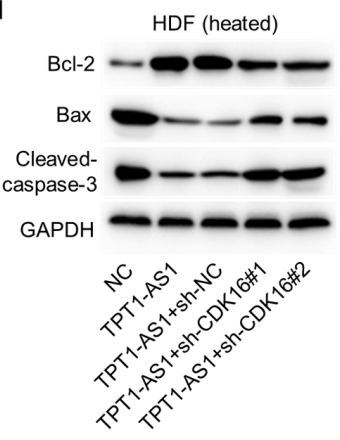

I

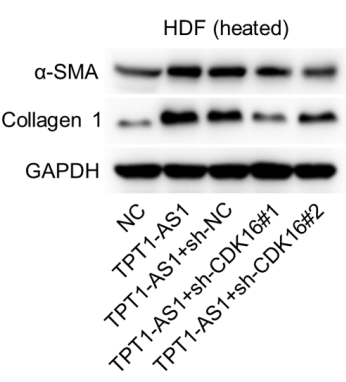

Figure 4. Silencing CDK16 abolishes the effects of TPT1-AS1 overexpression in thermally injured HDFs. (A) The silencing efficiency of CDK16 after the transfection of sh-CDK16\#1/\#2 in HDFs alone was detected by RT-qPCR analysis. (B) RT-qPCR was used to measure CDK16 expression in HDFs after the indicated treatment. (C) MTT confirmed the viability of the HDFs under the indicated treatment. (D) Flow cytometry was used to analyze cell cycle progression in each group. (E) Expression levels of cell cycle-associated markers were validated by western blot analysis after the indicated stimulation. (F and G) The apoptotic ability of HDFs under each stimulation was assessed via flow cytometric analysis. (H) Levels of apoptosis-associated proteins under the indicated treatments were evaluated by western blotting. (I) Western blot analysis was used to measure the levels of extracellular matrix synthesis-associated markers in each group. ${ }^{*} \mathrm{P}<0.05 ;{ }^{* *} \mathrm{P}<0.01 ;{ }^{*} \mathrm{P}<0.05$. TPT1-AS1, TPT1-antisense RNA 1; HDFs, human dermal fibroblasts; RT-qPCR, reverse transcription-quantitative PCR; sh-, short hairpin RNA-; NC, negative control; CDK16, cyclin-dependent kinase 16.

(Fig. 4F and G). In addition, the protein levels of Bax and cleaved caspase-3 were decreased, while the level of Bcl-2 was elevated by TPT1-AS1 overexpression in the heated HDFs; these outcomes were reversed by CDK16 knockdown 
(Fig. 4H). The promotive effect of upregulated TPT1-AS1 on ECM synthesis in the heat-stimulated HDFs was recovered after CDK16 downregulation (Fig. 4I).

\section{Discussion}

HDF cell function and ECM synthesis exert crucial impacts on wound healing after thermal injury. Collagen biosynthesis after injury is one of the major indicators for predicting the outcome of healing (18). Fibroblasts express and secrete $\alpha$-SMA protein during wound healing, which supports contraction. Collagen I and $\alpha$-SMA are two major indicators of ECM synthesis (19). In addition, HDF activation facilitates the restoration of tissue integrity (18). As previously reported, microarray analysis showed that TPT1-AS1 expression is downregulated in denatured dermal tissues (3). The present study initially demonstrated that TPT1-AS1 was significantly downregulated in the heat-stimulated HDFs. Gain-of-function assays showed that TPT1-AS1 promoted cell viability in the thermally injured HDFs. Moreover, the percentage of cells at each cell cycle stage indicated that TPT1-AS1 inhibited cell cycle arrest at the $G_{0} / G_{1}$ phase of heated HDFs. The levels of cell cycle-associated proteins (cyclin A1, cyclin D1, cyclin E1 and CDK4) were significantly decreased in the heated HDFs, an outcome that was reversed by TPT1-AS1 overexpression. TPT1-AS1 also decreased the cell apoptosis rate of the heated HDFs. The decrease in the protein levels of Bcl-2 or the increase in the levels of Bax and cleaved caspase-3 caused by thermal injury was reversed by the overexpression of TPT1-AS1. Furthermore, the increase in the protein levels of collagen I and $\alpha$-SMA induced by heat stimulation was rescued by TPT1-AS1 upregulation.

Moreover, the cytoplasmic localization of TPT1-AS1 defined its posttranscriptional regulation of gene expression. As previously reported, lncRNAs can serve as ceRNAs that post-transcriptionally regulate gene expression by competitively binding to miRNAs (20). Previous research has revealed that TPT1-AS1 serves as a decoy of certain miRNAs, such as miR-23a-5p (21) and miRNA-770-5p. Thus, it is proposed that the IncRNA TPT1-AS1, acting as a ceRNA, has a regulatory function by sponging miRNA and targeting mRNA in HDFs. Through a bioinformatics analysis, miR-324-5p was predicted to bind with TPT1-AS1. According to previous reports, miR-324-5p is upregulated in osteoarthritis (22) and hyperglycemia or hyperlipidemia (23). In the present study, the RT-qPCR analysis indicated that miR-324-5p was highly expressed in the heat-treated HDFs. Furthermore, a luciferase reporter assay revealed that TPT1-AS1 bound abundantly to miR-324-5p.

The evidence suggests that lncRNAs act as 'sponges' by competitively binding to common miRNAs, attenuating the repression of miRNAs on mRNAs (24-26). In order to verify the ceRNA regulatory network, the targets of miR-324-5p were identified. CDK16 was predicted as a putative target of miR-324-5p in the bioinformatics analysis. According to the literature, CDK16 has not been previously reported in diseases, including burn wounds. The present study found that CDK16 was expressed at low levels in HDFs after thermal injury. Mechanistically, CDK16 was targeted by miR-324-5p at positions 36-42 of the 3'UTR, and TPT1-AS1 upregulated
CDK16 expression by sponging miR-324-5p. The mRNA and protein levels of CDK16 were also negatively regulated by miR-324-5p in the HDFs. Functional interactions in ceRNA networks aid in coordinating multiple biological processes and, when perturbed, facilitate disease pathogenesis (27). Thus, a series of rescue assays were performed. The results indicated that CDK16 silencing reversed the promotion of TPT1-AS1 overexpression and cell viability and ECM synthesis, the suppression of cell cycle arrest at the $G_{0} / G_{1}$ phase and attenuated cell apoptosis. Thus, TPT1-AS1 contributes to cell viability, increases ECM synthesis, and inhibits cell cycle arrest and apoptosis to relieve the thermal injury of HDFs by upregulating CDK16 expression.

In summary, the present study is the first to demonstrate that TPT1-AS1 and CDK16 were upregulated in the HDFs after thermal injury and that miR-324-5p led to their significant downregulation in HDFs after thermal injury. TPT1-AS1 alleviated cell injury and induced ECM synthesis after thermal injury via the miR-324-5p/CDK16 axis. The present study suggested that TPT1-AS1 exerts a protective effect in the burn wound healing process. These findings may provide a novel target for burn wound therapy clinically.

\section{Acknowledgements}

Not applicable.

\section{Funding}

This study was supported by Science and Technology Program of Jiangxi, China (grant nos. 20171BBG70061 and S2020ZPY FB0615).

\section{Availability of data and materials}

The datasets used and/or analyzed during the current study are available from the corresponding author on reasonable request.

\section{Authors' contributions}

JL and HY performed the experiments and analyzed the data. $\mathrm{GG}, \mathrm{XC}$, and GZ helped to perform the experiments and analyzed the data. JL, HY, and JZ designed the study, wrote the manuscript and provided material support. JL, HY, and JZ confirmed the authenticity of all the raw data. All the authors read and approved the final manuscript.

\section{Ethics approval and consent to participate}

The use of HDFs was approved by the ethics committee of The First Affiliated Hospital of Nanchang University before the study (ethics approval no. 2020-016).

\section{Patient consent for publication}

Not applicable.

\section{Competing interests}

The authors declare that they have no competing interests. 


\section{References}

1. Zielins ER, Brett EA, Luan A, Hu MS, Walmsley GG, Paik K, Senarath-Yapa K, Atashroo DA, Wearda T, Lorenz HP, et al: Emerging drugs for the treatment of wound healing. Expert Opin Emerg Drugs 20: 235-246, 2015.

2. Cao W and Feng Y: LncRNA XIST promotes extracellular matrix synthesis, proliferation and migration by targeting miR-29b-3p/COL1A1 in human skin fibroblasts after thermal injury. Biol Res 52: 52, 2019.

3. Yu W, Guo Z, Liang P, Jiang B, Guo L, Duan M, Huang X, Zhang P, Zhang M, Ren L, et al: Expression changes in protein-coding genes and long non-coding RNAs in denatured dermis following thermal injury. Burns 46: 1128-1135, 2020.

4. Takeo M, Lee W and Ito M: Wound healing and skin regeneration. Cold Spring Harb Perspect Med 5: a023267, 2015.

5. Xue M and Jackson CJ: Extracellular matrix reorganization during wound healing and its impact on abnormal scarring. Adv Wound Care (New Rochelle) 4: 119-136, 2015.

6. Xu J, Bai J, Zhang X, Lv Y, Gong Y, Liu L, Zhao H, Yu F, Ping Y, Zhang $\mathrm{G}$, et al: A comprehensive overview of lncRNA annotation resources. Brief Bioinform 18: 236-249, 2017.

7. Zhang J, Cui X, Shen Y, Pang L, Zhang A, Fu Z, Chen J, Guo X, Gan W and Ji C: Distinct expression profiles of LncRNAs between brown adipose tissue and skeletal muscle. Biochem Biophys Res Commun 443: 1028-1034, 2014.

8. Li J, Long W, Li Q, Zhou Q, Wang Y, Wang H, Zhou B and Li J: Distinct expression profiles of lncRNAs between regressive and mature scars. Cell Physiol Biochem 35: 663-675, 2015.

9. Zheng $\mathrm{W}$ and $\mathrm{Yu} \mathrm{A}$ : EZH2-mediated suppression of lncRNA-LET promotes cell apoptosis and inhibits the proliferation of post-burn skin fibroblasts. Int J Mol Med 41: 1949-1957, 2018.

10. Chen L, Li J, Li Q, Li X, Gao Y, Hua X, Zhou B and Li J: Overexpression of LncRNA AC067945.2 down-regulates collagen expression in skin fibroblasts and possibly correlates with the VEGF and Wnt signalling pathways. Cell Physiol Biochem 45: 761-771, 2018.

11. Correia de Sousa M, Gjorgjieva M, Dolicka D, Sobolewski C and Foti M: Deciphering miRNAs' Action through miRNA Editing. Int J Mol Sci 20: 6249, 2019.

12. Lu TX and Rothenberg ME: MicroRNA. J Allergy Clin Immunol 141: 1202-1207, 2018.

13. Paraskevopoulou MD and Hatzigeorgiou AG: Analyzing miRNA-lncRNA interactions. Methods Mol Biol 1402: 271-286, 2016.

14. Yang S, Yin W, Ding Y and Liu F: Lnc RNA ZFAS1 regulates the proliferation, apoptosis, inflammatory response and autophagy of fibroblast-like synoviocytes via miR-2682-5p/ADAMTS9 axis in rheumatoid arthritis. Biosci Rep 40: BSR20201273, 2020.

15. Luo R, Jin H, Li L, Hu YX and Xiao F: Long noncoding RNA MEG3 inhibits apoptosis of retinal pigment epithelium cells induced by high glucose via the miR-93/Nrf2 Axis. Am J Pathol 190: 1813-1822, 2020.
16. Zhou S, Liang $P$, Zhang $P$, Zhang $M$ and Huang $X$ : The long noncoding RNA PDK1-AS/miR-125b-5p/VEGFA axis modulates human dermal microvascular endothelial cell and human umbilical vein endothelial cell angiogenesis after thermal injury. J Cell Physiol 236: 3129-3142, 2021.

17. Livak KJ and Schmittgen TD: Analysis of relative gene expression data using real-time quantitative PCR and the 2(-Delta Delta C(T)) method. Methods 25: 402-408, 2001.

18. Hinz B: Myofibroblasts. Exp Eye Res 142: 56-70, 2016.

19. Guo L, Huang X, Liang P, Zhang P, Zhang M, Ren L, Zeng J, Cui X and Huang X: Role of XIST/miR-29a/LIN28A pathway in denatured dermis and human skin fibroblasts (HSFs) after thermal injury. J Cell Biochem 119: 1463-1474, 2018.

20. Fu Z, Li G, Li Z, Wang Y, Zhao Y, Zheng S, Ye H, Luo Y, Zhao X, Wei L, et al: Endogenous miRNA Sponge LincRNAROR promotes proliferation, invasion and stem cell-like phenotype of pancreatic cancer cells. Cell Death Discov 3: 17004, 2017.

21. Gao X, Cao Y, Li J, Wang C and He H: LncRNA TPT1-AS1 Sponges miR-23a-5p in glioblastoma to promote cancer cell proliferation. Cancer Biother Radiopharm 2020 (Epub ahead of print).

22. Woods S, Barter MJ, Elliott HR, McGillivray CM, Birch MA, Clark IM and Young DA: miR-324-5p is up regulated in end-stage osteoarthritis and regulates Indian Hedgehog signalling by differing mechanisms in human and mouse. Matrix Biol 77: 87-100, 2019.

23. Guo J, Yang C, Lin Y, Hu G, Wei J, Zhang X, Chen X and Li J: Enhanced peripheral blood miR-324-5p is associated with the risk of metabolic syndrome by suppressing ROCK1. Biochim Biophys Acta Mol Cell Biol Lipids 1865: 158727, 2020.

24. An Y, Furber KL and Ji S: Pseudogenes regulate parental gene expression via ceRNA network. J Cell Mol Med 21: 185-192, 2017.

25. Liu XH, Sun M, Nie FQ, Ge YB, Zhang EB, Yin DD, Kong R, Xia R, Lu KH, Li JH, et al: Lnc RNA HOTAIR functions as a competing endogenous RNA to regulate HER 2 expression by sponging miR-331-3p in gastric cancer. Mol Cancer 13: 92, 2014.

26. Lei K, Liang X, Gao Y, Xu B, Xu Y, Li Y, Tao Y, Shi W and Liu J: Lnc-ATB contributes to gastric cancer growth through a miR-141-3p/TGF 32 feedback loop. Biochem Biophys Res Commun 484: 514-521, 2017.

27. Karreth FA and Pandolfi PP: ceRNA cross-talk in cancer: When ce-bling rivalries go awry. Cancer Discov 3: 1113-1121, 2013.

This work is licensed under a Creative Commons Attribution-NonCommercial-NoDerivatives 4.0 International (CC BY-NC-ND 4.0) License. 